\title{
Intuitionistic Fuzzy Nano Topological Space: Theory and Applications
}

\author{
M. Ramachandran' and A. Stephan Antony Raj ${ }^{2 *}$ \\ 'Assistant Professor, Department of Mathematics, Govt. Arts and Science College, Komarapalayam, Erode; \\ dr.ramachandran64@gmail.com \\ ${ }^{2}$ Assistant Professor, Department of Mathematics, SVS College of Engineering, Coimbatore; stephanraj138@gmail.com
}

\begin{abstract}
We introduce the notion of intuitionistic fuzzy nano topological space, its properties and results. The topological characterizations of intuitionistic fuzzy nano continuous functions are derived, and the weak forms of intuitionistic fuzzy nano-open sets are obtained. The intuitionistic fuzzy Nano Upper approximation space in real life application is discussed.
\end{abstract}

Keywords: Intuitionistic Fuzzy Nano Forms of Weakly Open Sets, Intuitionistic Fuzzy Nano Topological Space, Multi Criterion Decision Making

MSC (2010): Primary: 54B05, Secondary: 54C05, 08A72, 15B15, 68T37

\section{Introduction}

M. Lellis Thivagar and Carmel Richard[7-8] introduced nano topological space with respect to a subset $X$ of an universe $U$. We have investigated the notion of Intuitionistic Fuzzy nano topological space[2-3-4-5, 9-10-11-12-13-14$15-16,18]$.

\section{Intuitionistic Fuzzy Nano Topological Space}

\section{Definition 3.1}

Let $U$ be a non-empty, finite universe of objects and $R$ be an $I F$ equivalence relation on $U$. Let $\mathrm{A} \subseteq \mathrm{U}$. Let $\tau_{R}(X)=\left\{1 \sim, 0 \sim, I F L_{R}(X), I F U_{R}(X), I F B_{R}(X)\right\}$. Then $\tau_{R}(X)$ satisfies the axioms of topology. i.e., $\tau_{R}(X)$ is a topology on $U$ called the $I F$ nano topology on $U$ with respect to $A$. The elements of $\tau_{R}(X)$ are called as intu- itionistic fuzzy nano-open sets (IFNOS, for short). In this case, the pair $\left(U, \tau_{R}(X)\right)$ is called as intuitionistic fuzzy nano topological space (IFNTS, for short). In this regard, we refer[1,7-8,17].

\section{Proposition 2.2}

Let $(U, R)$ be an $I F$ approximation space (IFAS, for short), $C$ and $D$ subsets of $U$ then

$$
\begin{aligned}
& I F L_{R}(C) \subseteq C \subseteq I F U_{R}(C) \\
& I F L_{R}(0 \sim)=I F U_{R}(0 \sim)=0 \sim \\
& I F L_{R}(1 \sim)=I F U_{R}(1 \sim)=1 \sim \\
& I F U_{R}(C \cup D)=I F U_{R}(C) \cup I F U_{R}(D) \\
& I F U_{R}(C \cap D) \subseteq I F U_{R}(C) \cap I F U_{R}(D) \\
& I F L_{R}(C \cup D) \supseteq I F L_{R}(C) \cup I F L_{R}(D)
\end{aligned}
$$




$$
I F L_{R}(C \cap D)=I F L_{R}(C) \cap I F L_{R}(D)
$$

- $I F L_{R}(C) \subseteq I F L_{R}(D)$ and $I F U_{R}(C) \subseteq I F U_{R}(D)$ whenever $C \subseteq D$

- $\quad \operatorname{IFU}_{R}\left(C^{C}\right)=\left[\operatorname{IFL}_{R}(C)\right]^{C}$

- and $I F L_{R}\left(C^{C}\right)=\left[I F U_{R}(C)\right]^{C}$

$$
\begin{aligned}
& I F U_{R}\left(I F U_{R}(C)\right)=I F L_{R}(C) . \\
& I F L_{R}\left(I F U_{R}(C)\right)=I F U_{R}(C) .
\end{aligned}
$$

\section{Example 2.3}

Let $(U, R)$ be an IFAS where $U=\{d, e, f\}$ and $R \in R(U \times U)$ is defined as follows:

$R=\{\langle(d, d), 1 \sim, 0 \sim\rangle,\langle(d, e), 0.3,0.4\rangle,\langle(e, d), 0.3,0.4\rangle$, $\langle(e, e), 1 \sim, 0 \sim\rangle,\langle(e, f), 0.4,0.5\rangle,\langle(f, e), 0.4,0.5\rangle,\langle(f, f), 1 \sim$, $0 \sim\rangle,\langle(d, f), 0.4,0.3\rangle,\langle(f, d), 0.4,0.3\rangle\}$

Let $A=\{d, 0.7,0.3\rangle,\langle e, 0.6,0.4\rangle,\langle f, 0.6,0.4\rangle\}$ be an IFS on $U$ then by definition, we have

$$
\begin{aligned}
& I F U_{R}(A)=\left\{x, \mu_{I F U_{R}(A)}(x), v_{I F U_{R}(A)}(x) / x \in U\right\} \\
& I F L_{R}(A)=\left\{x, \mu_{I F L_{R}(A)}(x), v_{I F L_{R}(A)}(x) / x \in U\right\}
\end{aligned}
$$

Then,

$$
\begin{aligned}
\operatorname{IF~}_{\mathrm{R}}(\mathrm{A}) & =\{\langle\mathrm{d}, 0.7,0.3\rangle,\langle\mathrm{e}, 0.6,0.4\rangle,\langle\mathrm{f}, 0.6,0.3\rangle\} \\
\operatorname{IF~}_{\mathrm{R}}(\mathrm{A}) & =\{\langle\mathrm{d}, 0.6,0.4\rangle,\langle\mathrm{e}, 0.6,0.4\rangle,\langle\mathrm{f}, 0.6,0.4\rangle\} \\
\operatorname{IFB}_{\mathrm{R}}(\mathrm{A}) & =\{\langle\mathrm{d}, 0.4,0.6\rangle,\langle\mathrm{e}, 0.4,0.6\rangle,\langle\mathrm{f}, 0.4,0.6\rangle\} \\
\tau_{R}(X)=\{1 & \sim, 0 \sim, I_{R}(X), I_{R}\left(F U_{R}(X), I_{R}(X)\right\}
\end{aligned}
$$

\section{Remark 2.4 $4^{[1,8,17]}$}

Elements of $\left[\tau_{R}(A)\right]^{\mathcal{C}}$ are called IF nano closed sets (IFNCS, for short).

\section{Definition 2.5}

If $\left(U, \tau_{R}(X)\right)$ be an IFNTS in A, then the IF nano interior of $\mathrm{A}$ is defined as the union of all IFNOS contained in $\mathrm{A}$ and is denoted by $\operatorname{IFN} \operatorname{Int}(A)$.

i.e., $\operatorname{IFNInt}(A)=\cup\{G$ : $G$ is an IFNOS in $U$ and $G \subseteq A\}$. i.e., IFNInt $(A)$ is thelargest nano open subset of $\mathrm{A}[1,18,17]$.

\section{Definition 2.6}

The $I F$ nano closure of $A$ is defined as the intersection of all $I F$ closed subsets containing $A$ and is denoted by $\operatorname{IFNCl}(A)$.

i.e., $\operatorname{IFNCl}(A)=\cup\{K: K$ is an $I F N C S$ in $U$ and $A \subseteq K\}$.

i.e., $\operatorname{IFNCl}(A)$ is the smallest IFNCS containing $\mathrm{A}[1,18,17]$.

\section{Definition 2.7}

Let $\left(U, \tau_{\mathrm{R}}(X)\right)$ and $\left(V, \sigma_{\mathrm{R}}(Y)\right)$ be two IFNTSs. Then mapping $f: U \rightarrow V$ is an IF nano continuous (IFNC, for short) on $U$ if the inverse image of every IFNOS in $V$ is IFNOS in $U^{[1,8,17]}$

\section{Example 2.8}

Let $(U, R)$ be an IFAS where $U=\{a, b, c\}$ with $R=\{\langle(a, a), 1 \sim, 0 \sim\rangle,\langle(a, b), 0.3,0.3\rangle,\langle(b, a), 0.3,0.3\rangle$, $\langle(b, b), 1 \sim, 0 \sim\rangle$,

$\langle(b, c), 0.2,0.3\rangle, \quad\langle(c, b), 0.2,0.3\rangle, \quad\langle(c, c), 1 \sim, 0 \sim\rangle$, $\langle(a, c), 0.3,0.2\rangle, \quad\langle(c, a), 0.3,0.2\rangle\} . \quad\{\langle(\mathrm{a}, \mathrm{a}), 1 \sim, 0 \sim\rangle,\langle(\mathrm{a}, \mathrm{b}), 0.3$, $0.3\rangle,\langle(\mathrm{b}, \mathrm{a}), 0.3,0.3\rangle,\langle(\mathrm{b}, \mathrm{b}), 1 \sim, 0 \sim\rangle,\langle(\mathrm{b}, \mathrm{c}), 0.2,0.3\rangle,\langle(\mathrm{c}, \mathrm{b}), 0.2,0$. $3\rangle,\langle(\mathrm{c}, \mathrm{c}), 1 \sim, 0 \sim\rangle,\langle(\mathrm{a}, \mathrm{c}), 0.3,0.2\rangle,\langle(\mathrm{c}, \mathrm{a}), 0.3,0.2\rangle\}$

Let $X=\{\langle a, 0.2,0.1\rangle,\langle b, 0.2,0.3\rangle,\langle c, 0.3,0.2\rangle\}$ be an IFS on $U$ then

$\tau_{\mathrm{R}}(X)=\{1 \sim, 0 \sim,\{\langle a, 0.3,0.1\rangle,\langle b, 0.2,0.3\rangle,\langle c, 0.3$, $0.2\rangle\},\{\langle a, 0.2,0.3\rangle,\langle b, 0.2,0.3\rangle,\langle c, 0.2,0.3\rangle\},\{\langle a, 0.3,0.2\rangle$, $\langle b, 0.2,0.3\rangle,\langle c, 0.2,0.3\rangle\}$.

Let $(V, R)$ be an IFAS where $V=\{x, y, z\}$ with

$R=\{\langle(x, x), 1 \sim, 0 \sim\rangle,\langle(x, y), 0.5,0.4\rangle,\langle(y, x), 0.5,0.4\rangle$, $\langle(y, y), 1 \sim, 0 \sim\rangle$,

$\langle(y, z), 0.3,0.4\rangle,\langle(z, y), 0.3,0.4\rangle,\langle(z, z), 1 \sim, 0 \sim\rangle,\langle(x, z)$, $0.4,0.5\rangle,\langle(z, x), 0.4,0.5\rangle\}$.

Let $\mathrm{Y}=\{\langle x, 0.8,0.2\rangle,\langle y, 0.7,0.3\rangle,\langle z, 0.7,0.3\rangle\}$ be an IFS on $V$ then

$\sigma_{\mathrm{R}}(Y)=\{1 \sim, 0 \sim,\langle x, 0.8,0.2\rangle,\langle y, 0.7,0.3\rangle,\langle z, 0.7$, $0.3\rangle\},\{\langle x, 0.7,0.3\rangle,\langle y, 0.7,0.3\rangle,\langle z, 0.7,0.3\rangle\},\{\langle x, 0.3,0.7\rangle$, $\langle y, 0.3,0.7\rangle,\langle z, 0.3,0.7\rangle\}$.

Define $f:\left(U, \tau_{\mathrm{R}}(X)\right) \rightarrow\left(V, \sigma_{\mathrm{R}}(Y)\right)$ as $f(a)=x, f(b)=y, f(c)$ $=z$. Then the inverse image of every IFNOS in V is IFNOS in $U$.

\section{Definition 2.9}

A function $\mathrm{f}$ is said to be an $I F$ nano homeomorphism (IFNH, for short) if $f$ is

- $f$ is one-one and onto. 
- $f$ is IFNC.

- $f$ is IFNOS.

\section{Theorem 2.10}

Let $f:\left(U, \tau_{R}(X)\right) \rightarrow\left(V, \sigma_{R}(Y)\right)$ be a one-one onto mapping, then $f$ is IFNH if and only if $f$ is IF nano closed and IFNC.

\section{Proof}

Let $f$ be a IFNH. Then $f$ is IFNC. Let $S$ be an arbitrary IFNCS in $\left(U, \tau_{R}(X)\right)$. Then $U-S$ is IFNOS. Since $f$ is IFNOS, $f(U-S)$ is IFNOS in $V$. That is, $V-f(S)$ is IFNOS in $V$. Therefore, $f(S)$ is $I F$ nano closed in $V$. Thus, the image of every IFNCS in $U$ is IFNCS in $V$. That is, $f$ is IFNCS. Conversely, let $f$ be IF nano closed and IFNC. Let B be IFNOS in $\left(U, \tau_{R}(X)\right)$. Then $U-B$ is IFNCS in $U$. Since $f$ is IF nano closed, $f(U-B)$ $=V-f(B)$ is $I F$ nano closed in $V$. Therefore, $f(B)$ is IFNOS in V. Thus, $f$ is IFNOS and hence $f$ is a IFNH.

\section{Theorem 2.11}

A one-one function $f$ of $(U, \tau R(X))$ onto $(V, \sigma R(Y))$ is a IFNH if and only if $f(\operatorname{IFNCl}(A))=\operatorname{IFNCl}(f(A))$ for every subset $A$ of $U$.

\section{Proof}

If $f$ is an IFNH, $f$ is IFNC and $I F$ nano closed. If $A \subseteq U$, $f(I F N C l(A)) \subseteq I F N C l(f(A))$ since $f$ is an IFNC. Since IFNCl(A) is an $I F$ nano closed in $U$ and $f$ is IF nano closed, $f(I F N C l(A))$ is IF nano closed in $V$. Therefore $\operatorname{IFNCl(f(IFNCl(A)))=}$ $f(\operatorname{IFNCl}(A))$. Since $A \subseteq \operatorname{IFNCl}(A), f(A) \subseteq f(\operatorname{IFNCl}(A))$ and

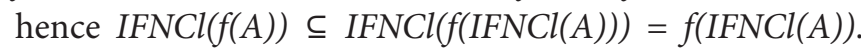
Thus IFNCl(f(A)) $=f(\operatorname{IFNCl}(A))$ if $f$ is IFNH. Conversely, if $\operatorname{IFNCl}(f(A))=f(\operatorname{IFNCl}(A))$ for every subset $A$ of $U$, then $f$ is IFNC. If $A$ is IF nano closed in $U, A=\operatorname{IFNCl}(A)$ which implies $f(A)=f(\operatorname{IFNCl}(A))$. Therefore, $\operatorname{IFNCl}(f(A))=f(A)$. Thus, $f(A)$ is an IF nano closed. Also $f$ is IFNC. Therefore $f$ is an IFNH.

\section{IF Nano Forms of Weakly Open Sets}

Let $\left(U, \tau_{R}(X)\right)$ be an $I F$ nano topological space (IFNTS) with respect to $X$ where $X \subseteq U, R$ is an equivalence relation on $U$.

$\frac{U}{R}$ denotes the family of equivalence classes of $U$ by $R$.

\section{Definition 3.1}

Let $\left(U, \tau_{R}(X)\right)$ be an IFNTS and $B \subseteq U$. Then $B$ is said to be - IF nano semi-open if
$B \subseteq \operatorname{IFNCl(IFNInt(B))}$

- $\quad I F$ nano pre-open if $B \subseteq \operatorname{IFNInt}(\operatorname{IFNCl}(B))$

- IF nano a-open if $B \subseteq \operatorname{IFNInt}(\operatorname{IFNCl}(\operatorname{IFNInt}(B)))$

- IF regular open if $B=\operatorname{IFN} \operatorname{Int}(\operatorname{IFNCl}(B))$

IFNSO, IFNPO, IFNa-open and IFRO respectively denote the families of all IFNSO, IF nano pre-open, IF nano a-open and IF regular open subsets of $U$.

\section{Example 3.2}

Let $(U, R)$ be an IFAS where $U=\{a, b, c\}$ and $R \in R(U \times U)$ is defined as follows:

$R=\{\langle(a, a), 1 \sim, 0 \sim\rangle,\langle(a, b), 0.3,0.4\rangle,\langle(b, a), 0.3,0.4\rangle,\langle(b$, $b), 1 \sim, 0 \sim\rangle,\langle(b, c), 0.4,0.5\rangle,\langle(c, b), 0.4,0.5\rangle,\langle(c, c), 1 \sim, 0 \sim\rangle$, $\langle(a, c), 0.4,0.3\rangle,\langle(c, a), 0.4,0.3\rangle\}$.

Let $X=\{\langle a, 0.7,0.3\rangle,\langle b, 0.6,0.4\rangle,\langle c, 0.6,0.4\rangle\}$ be an $I F$ set on $U$ then by definition, we have

$\tau_{R}(A)=\{1 \sim, 0 \sim,\{\langle a, 0.7,0.3\rangle,\langle b, 0.6,0.4\rangle,\langle c, 0.6,0.3\rangle\}$,

$\{\langle\mathrm{a}, 0.6,0.4\rangle,\langle\mathrm{b}, 0.6,0.4\rangle,\langle\mathrm{c}, 0.6,0.4\rangle\},\{\langle\mathrm{a}, 0.4,0.6\rangle,\langle\mathrm{b}, 0.4,0.6\rangle$, $\langle c, 0.4,0.6\rangle\}\}$.

Let $A=\{\langle a, 0.7,0.3\rangle,\langle b, 0.6,0.4\rangle,\langle c, 0.6,0.3\rangle\}$, then the IFSA is an IFNSO in $U$.

Let $A=\{\langle a, 0.6,0.4\rangle,\langle b, 0.6,0.4\rangle,\langle c, 0.6,0.4\rangle\}$, then the IFSA is an IFNPO in $U$.

Let $A=\{\langle a, 0.7,0.3\rangle,\langle b, 0.6,0.4\rangle,\langle c, 0.6,0.3\rangle\}$, then the IFSA is an IFNa-open in $U$.

Let $A=\{\langle a, 0.6,0.4\rangle,\langle b, 0.6,0.4\rangle,\langle c, 0.6,0.4\rangle\}$, then the IFSA is an IFNRO in $U$.

\section{Theorem 3.3}

If $A$ is $I F N O$ in $\left(U, \tau_{R}(X)\right)$, then it is IFNa-open in $U$.

\section{Proof}

Since $\mathrm{A}$ is $\operatorname{IFNO}$ in $\mathrm{U}, \operatorname{IFNInt}(A)=A$.

Then $\operatorname{IFNCl}(\operatorname{IFNInt}(A))=\operatorname{IFNCl}(A) \supseteq A$. That is, $A \subset \operatorname{IFNCl}(\operatorname{IFNInt}(A))$. Therefore, IFNInt (A) $\subseteq \operatorname{IFNInt}(\operatorname{IFNCl}(\operatorname{IFNInt}(A)))$. That is, $A \subseteq I F N I n t$ (IFNCl(IFNInt(A))). Thus, A is IFNa-open.

\section{Theorem 3.4}

$\tau_{R}^{\alpha}(X) \subseteq \operatorname{IFNSO}(U, X)$ in a $\operatorname{IFNTS}(U, \tau R(X))$.

\section{Proof}

If $A \in \tau_{R}^{\alpha}(X), A \subseteq \operatorname{IFNInt(IFNCl(IFNInt(A)))\subseteq }$

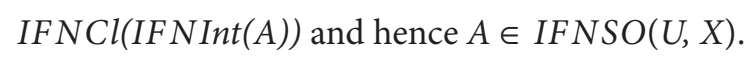




\section{Theorem 3.5}

$\tau_{R}^{\alpha}(X) \subseteq \operatorname{IFNSO}(U, X)$ in a $\operatorname{IFNTS}\left(U, \tau_{R}(X)\right)$.

\section{Proof}

If $A \in \tau_{R}^{\alpha}(X), A \subseteq \operatorname{IFNInt}(\operatorname{IFNCl}(\operatorname{IFNInt}(A)))$. Since $\operatorname{IFNInt}(A) \subseteq \mathrm{A},, \operatorname{IFNInt}(\operatorname{IFNCl}(\operatorname{IFNInt}(A))) \subseteq \operatorname{IFNInt}$ $(\operatorname{IFNCl}(A))$. That is, $A \subseteq \operatorname{IFNInt}(\operatorname{IFNCl}(A))$. That is, $\tau_{R}^{\alpha}$ $(X) \subseteq \operatorname{IFNPO}(U, X)$.

\section{Theorem 3.6}

If, in a $\operatorname{IFNTS}(U, \tau R(X)), I F L_{R}(X)=I F U_{R}(X)=X$, then $1 \sim, 0 \sim, I F L_{R}(X)\left(=I F U_{R}(X)\right)$ and any set $A \supset$ $\operatorname{IF} L(X)$ are the only IFNa-open sets in $U$.

\section{Proof}

Since $I F L_{R}(X)=I F U_{R}(X)=X$, the $I F$ nano topology, $\tau_{R}(X)=\left\{1 \sim, 0 \sim, I F L_{R}(X)\right\}$. Since any IFNOS is IF nano a-open, $1 \sim, O \sim$ and $I F L_{R}(X)$ are $I F$ nano $\alpha$-open in U. If $A \subset I F L_{R}(X)$, then $\operatorname{IFN} \operatorname{Int}(A)=O \sim$, since $O \sim$ is the only IFNO subset of $A$. Therefore $\operatorname{IFNCl(IFNInt}(A))=$ $O \sim$ and hence $A$ is not $I F$ nano $\alpha$-open. If $A \supset$ $I F L_{R}(X), \operatorname{IFL}_{\mathrm{R}}(\mathrm{X})$ is the largest IFNO subset of $A$ and hence, IFNInt $\operatorname{IFNCl(IFNInt}(A)))=$ $\operatorname{IFN} \operatorname{Int}\left(\operatorname{IFNCl}\left(L_{R}(X)\right)\right)=\operatorname{IFNInt}\left(B_{R}(X)^{c}\right)=$ $\operatorname{IFN} \operatorname{Int}(U)$, Since $\operatorname{IF} B_{R}(X)=0 \sim$. Therefore, $\operatorname{IFNInt}(\operatorname{IFNCl}(\operatorname{IFNInt}(A)))=U$ and hence, $A \subseteq$ IFNInt(IFNCl(IFNInt(A))). Therefore, $A$ is $I F N a$-open. Thus $U, O \sim, I F L_{R}(X)$ and any set $A$ $\supset I F L_{R}(X)$ are the only $I F N \alpha$-open sets in $U$, if $I F L_{R}(X)=I F U_{R}(X)$.

\section{Theorem 3.7}

$1 \sim, 0 \sim, I F U_{R}(X)$ and any set $A \supset I F U_{R}(X)$ are the only $\operatorname{IFNa-open~sets~in~a~} \operatorname{IFNTS}\left(U, \tau_{R}(X)\right)$, if $\operatorname{IFL}_{R}(X)=$ $0 \sim$.

\section{Proof}

Since $\operatorname{IFL}_{R}(X)=0 \sim, \operatorname{IFB}_{R}(X)=\operatorname{IFU}_{R}(X)$.. Therefore, $\tau_{R}(X)=\left\{1 \sim, O \sim, I F U_{R}(X)\right\}$ and the members of $\tau_{R}(X)$ are IFNa-open in $U$. Let $A \subset$ $\operatorname{IFU}_{R}(X)$. Then $\operatorname{IFNInt}(A)=0 \sim$ and hence $\operatorname{IFNInt}(\operatorname{IFNCl}(\operatorname{IFNInt}(A)))=0 \sim$. Therefore $A$ is not $I F N a-o p e n$ in $U$. If $A \supset \operatorname{IFU}_{R}(X)$, then $\operatorname{IFU}_{R}(X)$ is the largest $I F N a$-open subset of $A$ (unless, $I F U_{R}(X)=U$, in case of which $1 \sim$ and $O \sim$ are the only nano-open sets in $U)$. Therefore, $\operatorname{IFNInt}(\operatorname{IFNCl}(\operatorname{IFNInt}(A)))=$ $\operatorname{IFNInt}\left(\operatorname{IFNCl}\left(\operatorname{IFU}_{R}(X)\right)\right)=\operatorname{IFNInt}(U)$ and hence

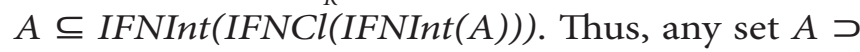
$U_{R}(X)$ is IFNa-open in $U$. Hence, $1 \sim, 0 \sim, \operatorname{IFU}_{R}(X)$ and any superset of $\operatorname{IFU}_{R}(X)$ are the only $I F N \alpha$-open sets in $U$.

Corollary $3.8 \tau_{R}(X)=\tau_{R}^{\alpha}(X)$, if $I F U_{R}(X)=U$.

\section{Theorem 3.9}

If, in a $\operatorname{IFNTS}\left(\mathrm{U}_{,} \tau_{\mathrm{R}}(\mathrm{X})\right), \operatorname{IFU}_{\mathrm{R}}(\mathrm{X})=\mathrm{IFL}_{\mathrm{R}}(\mathrm{X})$, then $0 \sim$ and set $\mathrm{A}$ such that $\mathrm{A} \supseteq \operatorname{IFL}_{\mathrm{R}}(\mathrm{X})$ are the only IFNaopen subsets of $U$.

\section{Proof}

$\tau_{R}(X)=\left\{1 \sim, O \sim, I F L_{R}(X)\right\} .0 \sim$ is IFNa-open. If $A$ is an non-empty subset of $U$ and $A \subset I F L_{R}(X)$, then $\operatorname{IFNCl}(\operatorname{IFNInt}(A))=\operatorname{IFNCl}(O \sim)=0 \sim$. Therefore, A is not $I F N a$-open, if $A \subset I F L_{R}(X)$. If $A \supseteq I F L_{R}(X)$, then $\operatorname{IFNCl}(\operatorname{IFNInt}(A))=\operatorname{IFNCl}\left(\operatorname{IFL}_{R}(X)\right)=$ $U$, since $\operatorname{IFU}_{R}(X)=\operatorname{IFU}_{R}(X)$. Therefore, $A \subseteq$ $\operatorname{IFNCl}(\operatorname{IFNInt}(A))$ and hence $A$ is IFNa-open. Thus $O \sim$ and sets containing $I F L_{R}(X)$ are the only $I F N \alpha-$ open sets in $U$, if $\operatorname{IFU}_{R}(X)=\operatorname{IFL}_{R}(X)$.

\section{Theorem 3.10}

Any IFRO set is IFNO.

\section{Proof}

If $A$ is $\operatorname{IFRO}$ in $\left(U, \tau_{R}(X)\right), A=\operatorname{IFNInt}(\operatorname{IFNCl}(A))$. Then $\operatorname{IFN} \operatorname{Int}(A)=\operatorname{IFN} \operatorname{Int}(\operatorname{IFN} \operatorname{Int}(\operatorname{IFNCl}(A)))$ $=A$. That is, $A$ is IFNO in $U$.

\section{Remark 3.11}

The converse of the above theorem is not true. For example, let $(U, R)$ be an $I F$ approximation space where $U=\{a, b, c\}$ with

$$
\mathrm{R}=\{\langle(\mathrm{a}, \mathrm{a}), 1 \sim, 0 \sim\rangle,\langle(\mathrm{a}, \mathrm{b}), 0.3,0.3\rangle,\langle(\mathrm{b}, \mathrm{a}), 0.3,0.3\rangle,
$$
$\langle(\mathrm{b}, \mathrm{b}), 1 \sim, 0 \sim\rangle,\langle(\mathrm{b}, \mathrm{c}), 0.2,0.3\rangle,\langle(\mathrm{c}, \mathrm{b}), 0.2,0.3\rangle,\langle(\mathrm{c}, \mathrm{c}), 1 \sim, 0 \sim\rangle$, $\langle(\mathrm{a}, \mathrm{c}), 0.3,0.2\rangle,\langle(\mathrm{c}, \mathrm{a}), 0.3,0.2\rangle\}$.

Let $X=\{\langle a, 0.2,0.1\rangle,\langle b, 0.2,0.3\rangle,\langle c, 0.3,0.2\rangle\}$ be an IF set on $U$ then

$$
\tau_{R}(X)=\{1 \sim, 0 \sim,\{\langle a, 0.3,0.1\rangle,\langle b, 0.2,0.3\rangle,\langle c, 0.3,0.2\rangle\},
$$
$\{\langle a, 0.2,0.3\rangle,\langle b, 0.2,0.3\rangle,\langle c, 0.2,0.3\rangle\},\{\langle a, 0.3,0.2\rangle,\langle b, 0.2,0.3\rangle$, $\langle c, 0.2,0.3\rangle\}\}$. 
Let $A=\{\langle a, 0.3,0.1\rangle,\langle b, 0.2,0.3\rangle,\langle c, 0.3,0.2\rangle\}$ and $B=$ $\{\langle a, 0.2,0.2\rangle,\langle b, 0.2,0.2\rangle,\langle c, 0.2,0.2\rangle\}$ be IFRO sets then $A \cup B$ $=\{\langle a, 0.3,0.1\rangle,\langle b, 0.2,0.2\rangle,\langle c, 0.3,0.2\rangle\}$ is not IFRO set.

\section{Theorem 3.12}

In a $\operatorname{IFNTS}\left(U, \tau_{R}(X)\right)$, if $\operatorname{IF} U_{R}(X) \neq I F L_{R}(X)$, then the only IF $R O$ sets are $1 \sim, 0 \sim, I F L_{R}(X)$ and $I F B_{R}(X)$.

\section{Proof}

The only IFNOSs in $\left(U, \tau_{R}(X)\right)$ are $1 \sim, 0 \sim, I F L_{R}(X)$, $I F U_{R}(X)$ and $I F B_{R}(X)$ and hence the only IFCO sets in $U$ are $1 \sim, 0 \sim,\left[I F L_{R}(X)\right]^{C},\left[I F U_{R}(X)\right]^{c}$ and $\left[I F B_{R}(X)\right]^{C}$.

\section{Case 1}

Let $A=\operatorname{IFBR}(X)$. Then $\operatorname{IFNCl}(A)=\left[\operatorname{IFB}_{R}(X)\right]^{c}$. Therefore, $\operatorname{IFNInt}(\operatorname{IFNCl}(A))=\operatorname{IFNInt}\left[\operatorname{IFB}_{R}(X)\right]^{C}$ $=\left[\operatorname{IFNCl}\left(\operatorname{IFB}_{R}(X)\right)\right]^{\mathcal{C}}=\left[\left(\operatorname{IFL}_{R}(X)\right)^{\mathcal{C}}\right]^{\mathcal{C}}=\operatorname{IFL}_{R}(X)=$ $A$. Therefore, $A=I F L_{R}(X)$ is IFRO.

\section{Case 2}

Let $A=\operatorname{IF} B_{R}(X)$. Then $\operatorname{IFNCl}(A)=\left[I F L_{R}(X)\right]^{C}$. Therefore, IFNInt $(\operatorname{IFNCl}(A))=\operatorname{IFNInt}\left[\operatorname{IF} L_{R}(X)\right]^{C}=$ $\left[\operatorname{IFNCl}\left(\operatorname{IFL}_{R}(X)\right)\right]^{c}$

$=\left[\left(I F B_{R}(X)\right)^{c}\right]^{c}=I F B_{R}(X)=A$. Therefore, $A=$ $\operatorname{IFB}_{R}(X)$ is IFRO.

\section{Case 3}

Let $A=I F U_{R}(X)$. Then $\operatorname{IFNCl}(A)=U$. Therefore, $\operatorname{IFNInt}(\operatorname{IFNCl}(A))=\operatorname{IFN} \operatorname{Int}(U)=U \neq A$. That is, $\operatorname{IF} U R(X)=A$ is not $I F R O$ unless $I F U_{R}(X)=U$.

\section{Case 4}

Since IFNInt $(\operatorname{IFNCl}(A))=\operatorname{IFNInt}(0 \sim)=0 \sim, 1 \sim$ and $0 \sim$ are IFRO. Also any IFRO is IFNO. Thus, $1 \sim, 0 \sim$, $I F L_{R}(X)$ and $I F B_{R}(X)$ are the only IFRO sets.

\section{A Real Life Application}

We discuss a real life application of IF NTS on one or more universal sets to multi criterion decision making using IFNUAS. It is observed that in the case of insurance companies by investors due to various factors like affordable premium, quality of service, quaranteed returns, location of the company and various best products, investors depend on one or more insurance companies. Hence, IF relation provides the better relation between the investors and insurance companies.

Consider $V=\left\{v_{1}, v_{2}, v_{3}, v_{4}, v_{5}\right\}$, in which $v_{1}$ is affordable premium; $v_{2}$ is quality of service; $v_{3}$ is quaran- teed returns; $v_{4}$ is location of the company; $v_{5}$ is various best products and decisions $U=\left\{u_{1}, u_{2}, u_{3}, u_{4}, u_{5}\right\}$, in which $u_{1}$ is excellent; $u_{2}$ is good; $u_{3}$ is satisfactory; $u_{4}$ is acceptable; $u_{5}$ is least acceptable. Investors from various financial status are invited to the survey. Therefore, $(U, V$, $\left.I F U_{R}, I F L_{R}\right)$ be an IFAS, where $U=\left\{u_{1}, u_{2}, u_{3}, u_{4}, u_{5}\right\}$ and $V=\left\{v_{1}, v_{2}, v_{3}, v_{4}, v_{5}\right\}$.

If $16 \%$ investors give excellent and $11 \%$ give not excellent; $26 \%$ give good; $21 \%$ give not good; $36 \%$ give satisfactory; $6 \%$ give not satisfactory; $11 \%$ give acceptable; $22 \%$ give not acceptable; $16 \%$ give least acceptable and $10 \%$ give not acceptable, then we have $(.16, .11 ; .26, .21 ; .36, .06$; $.11, .22 ; .16, .1)^{t}$. Similarly, for other criterias: $(.56, .2 ; .16$, $.46 ; .3, .16 ; 0, .6 ; .3, .7)^{t},(.21, .3 ; .36, .22 ; .26, .16 ; .2, .7 ; .2, .3)$ t, $(.1, .8 ; .2, .5 ; .5, .3 ; .3, .4 ; .3, .1)^{t}$ and $(0, .7 ; 0, .6 ; .16, .4 ; .3$, $.6, .2 ; .6, .15)^{t}$. Based on the decision vectors, the IF relation from $\mathrm{U}$ to $\mathrm{V}$ is given by the following matrix.

\begin{tabular}{|c|c|c|c|c|c|}
\hline & $V_{1}$ & $V_{2}$ & $V_{3}$ & $V_{4}$ & $V_{5}$ \\
\hline 1 & $\langle 0.16,0.11\rangle$ & $\langle 0.56,0.02\rangle$ & $\langle 0.21,0.03\rangle$ & $\langle 0.01,0.08\rangle$ & $\langle 0.00,0.07\rangle$ \\
\hline$u_{2}$ & $\langle 0.26,0.21\rangle$ & $\langle 0.16,0.46\rangle$ & $\langle 0.36,0.22\rangle$ & $\langle 0.02,0.05\rangle$ & $\langle 0.00,0.06\rangle$ \\
\hline & $\langle 0.36,0.06\rangle$ & $\langle 0.03,0.16\rangle$ & $\langle 0.26,0.16\rangle$ & $\langle 0.05,0.03\rangle$ & $\langle 0.16,0.04\rangle$ \\
\hline$u_{4}$ & $\langle 0.11,0.22\rangle$ & $\langle 0.00,0.06\rangle$ & $\langle 0.02,0.07\rangle$ & $\langle 0.03,0.04\rangle$ & $\langle 0.36,0.02\rangle$ \\
\hline & $\langle 0.16,0.01\rangle$ & $\langle 0.03,0.07\rangle$ & $\langle 0.03,0.07\rangle$ & $\langle 0.03,0.01\rangle$ & $\langle 0.06,0.15\rangle$ \\
\hline
\end{tabular}

Two category of investors are considered, where right weightage for each criterion in $U$ are $U_{1}=\left(\left\langle u_{1}, .36, .15\right\rangle\right.$, $\left.\left\langle u_{2}, 16, .3\right\rangle,\left\langle u_{3^{3}}, 3, .3\right\rangle,\left\langle u_{4}, 2,0.5\right\rangle,\left\langle u_{5^{\prime}}, 1, .4\right\rangle\right)$ and $U_{2}=\left(\left\langle u_{1}, 0.21,0.32\right\rangle,\left\langle u_{2}, 0.16,0.42\right\rangle,\left\langle u_{3}, 0.2,0.4\right\rangle\right.$, $\left.\left.<u_{4}, 0.2,0.4\right\rangle,\left\langle u_{5}, 0.2,0.3\right\rangle\right)$ respectively. Thus, by using IF upper approximation we have:

$I F U_{R}\left(V_{1}\right)=\left(\left\langle u_{1}, 0.21,0.15\right\rangle,\left\langle u_{2}, 0.3,0.21\right\rangle\right.$, $\left\langle u_{3}, 0.36,0.15\right\rangle,\left\langle u_{4}, 0.3,0.22\right\rangle,\left\langle u_{5}, 0.2,0.15>\right)^{t}$ and $I_{R} U_{R}\left(V_{2}\right)=\left(\left\langle u_{1}, 0.21,0.3\right\rangle,\left\langle u_{2}, 0.36,0.22\right\rangle\right.$, $\left.<u_{3}, 0.26,0.2>, \quad<u_{4}, 0.2,0.3>, \quad<u_{5}, 0.2,0.3>\right)^{\mathrm{t}}$ respectively.

From above, according to the principle of maximum membership, the decision for the first category of investors is satisfactory whereas for the second category it is good.

\section{Conclusion}

The main research is focused on introducing intuitionistic fuzzy nano topological space with some properties and its characterizations. We have investigated a real time problem in Multi Criterion Decision Making. 


\section{References}

1. Atanassov KT. Intuitionistic Fuzzy Sets. Fuzzy Sets and Systems. 1986; 20(1): 87-96. https://doi.org/10.1016/S01650114(86)80034-3

2. Atanassov KT. Review and new result on IF Set, Mathematical foundation of artificial intelligence seminar, Sofia, Preprint I-88, 1988.

3. Atanassov KT. More on IF Sets. Fuzzy Sets and Systems. 1989; 33(1): 37-45. https://doi.org/10.1016/0165-0114(89)90215-7

4. Atanassov KT. New operations defined over the IF Sets. Fuzzy Sets and Systems. 1994; 61: 137-142. https://doi. org/10.1016/0165-0114(94)90229-1

5. Coker D. An Introduction to IF Topological Spaces. Fuzzy Sets and Systems. 1997; 88: 81-9. https://doi.org/10.1016/S01650114(96)00076-0

6. Cornelis C, Cock MD, Kerre EE. IF rough sets: At the crossroads of imperfect knowledge, Expert Syst. 2003; 20: 260-70. https://doi.org/10.1111/1468-0394.00250

7. Hanafy IM, El A. Completely continuous functions in IF topological spaces. Czechoslovak Mathematical Journal. 2003; 53: 793-803. https://doi.org/10.1023/ B:CMAJ.0000024523.64828.31

8. Lellis Thivagar M, Richard C. On Nano Continuity. Mathematical Theory and Modelling. 2013; 3(7): 32-7.

9. Lellis Thivagar M, Richard C. On Nano Forms of Weakly Open Sets. International Journal of Mathematics and Statistics Invention. 2013; 1(1): 31-7.

10. Lin TY. Granular computing: Fuzzy logic and rough sets. In: Zadeh LA, Kacprzk, Editors . Computing with words in information/intelligent theories. Int. J. Information Comput. Sci. 1985; 11(5): 99-102.

11. Pawlak Z. Rough sets. International Journal of Computer and Information Sciences. 1982; 11: 341-56. https://doi. org/10.1007/BF01001956

12. Pawlak Z. Rough sets - Theoretical Aspects of Reasoning about data. Boston: Kluwer Academic Publishers; 1991.

13. Pawlak Z. Rough set theory and its applications. Journal of Telecommunications and Information Technology. 2002; 3: 7-10.

14. Pawlak Z., Zdzislaw. Rough set approach to knowledgebased decision support. European Journal of Operational Research. 1996; 99(1): 48-57. https://doi.org/10.1016/S03772217(96)00382-7

15. Pawlak Z., Zdzislaw. Rough set approach to Multi-attribute decision analysis. European Journal of Operational Research. 1997; 72(3): 443-53. https://doi.org/10.1016/03772217(94)90415-4

16. Tripathy BK. Rough sets on IF Approximation Spaces. In: Proceedings of 3rd International IEEE Conference on Intelligent Systems (IS06). p. 776-79. PMid:26029348 PMCid:PMC4444617

17. Wu, W-Z, Zhou L. On IF topologies based on IF reflexive and transitive relations. Soft Comput. 2011; 15: 1183-94. https:// doi.org/10.1007/s00500-010-0576-0

18. Zadeh LA. Fuzzy sets. Information and Control. 1965; 8(3): 338-53. https://doi.org/10.1016/S0019-9958(65)90241-X 